\title{
Constraints on the Equation of State of Dense Nuclear Matter from the Tidal Deformability of Neutron Stars
}

\author{
A. Kanakis-Pegios, P.S. Koliogiannis, Ch.C. Moustakidis \\ Department of Theoretical Physics, Aristotle University of Thessaloniki, \\ 54124 Thessaloniki, Greece
}

Received 20 October 2021

doi: https://doi.org/10.55318/bgjp.2021.48.5-6.577

\begin{abstract}
Neutron stars constitute a very promising natural laboratory for studying the properties of dense nuclear matter and the equation of state. One microscopic parameter that is of great interest is the speed of sound, especially the upper bound on it. This work is based on the idea to examine possible constraints on the speed of sound by using neutron stars. For this purpose, in our study, we use the observed effective tidal deformability from binary neutron star systems as a tool to impose constraints on the equation of state through the upper bound on the speed of sound. In our approach, we parametrize the stiffness of the equation of state by using the speed of sound, for various transition density values. The two recent observations of binary neutron star mergers from LIGO/VIRGO have been used to impose robust constraints. Furthermore, we extended our study in the hypothetical scenario of a very massive neutron star by using the recent observation of the GW190814 event. The possibility of the existence of such a massive non-rotating neutron star cannot be excluded according to our study. Furthermore, we postulate what kind of future observations would be useful to impose more stringent constraints on the properties of the equation of state.
\end{abstract}

KEY WORDS: equation of state, gravitational waves, neutron stars, speed of sound, tidal deformability.

\section{Introduction}

The properties of dense nuclear matter are currently an unsolved problem in Nuclear Physics. One of the most promising tools in nature to study the dense nuclear matter are the neutron stars [1]. The quantity of the speed of sound is of great interest, especially its upper bound, since it affects the equation of state (EoS).

Regarding the upper bound of the speed of sound, the main assumption is that fulfills the causality, i.e. the speed of sound cannot exceed the speed of light [2]. 
Various studies have been made about the upper bound on the speed of sound [3, 4]. Moreover, in Ref. [4] the authors found also that the existence of neutron stars with masses about two solar masses, in combination with the knowledge of the EoS of hadronic matter at the low density region, is not consistent with the value $c / \sqrt{3}$ for the bound of the speed of sound. The effects of the speed of sound on the tidal deformability of neutron stars have also been studied in Refs. [5-8].

We underline the two main considerations for the possible upper bound in the speed of sound of dense nuclear matter; (a) the causality which leads to an upper bound on the speed of sound $v_{s}=c$, and (b) the conformal upper limit $v_{s}=$ $c / \sqrt{3}$. To be more specific, the conformal limit $v_{s}=c / \sqrt{3}$ is related to the existence of quark matter in the neutron star interior. Therefore, the study of this suggested case of the speed of sound bound is very important (see also Refs. $[9,10])$

At the core of our work lies the idea of using the tidal deformability of neutron stars to obtain possible constraints on the speed of sound and its upper bounds. We notice the arising contradiction regarding the stiffness of the EoS; the recent high measurements of neutron star masses favor stiff EoS, while the observed upper limit on the effective tidal deformability (from gravitational-wave detectors) favors softer EoSs. Our study aims to shed more light on that, by parametrizing the stiffness of the EoS through the upper bounds of the speed of sound and the transition density. The predictions are combined mainly with the recent observations of two events, GW170817 and GW190425 [11-14].

The demand of a) a soft EoS at the low density region (in order to be in accordance with the upper limit of the tidal deformability) and b) a stiff EoS for high densities (in order to predict the high neutron star masses) leads to robust constraints on the EoS. Additionally, we postulate what kind of future events could be helpful. For more details, we refer to the original study [15].

Moreover, we mention the recent detection of GW190814 event [16], in which the lighter component compact object might be a neutron star. Following the hypothesis of the existence of such a massive non-rotating neutron star, we extended our work by studying the role of the speed of sound in that case [17].

\section{Theoretical Framework}

\subsection{Speed of sound and EoS approach}

Firstly, the maximum mass configuration that we used, by parametrizing the neutron star EoS is given below [18]

$$
P(\mathcal{E})= \begin{cases}P_{\text {crust }}(\mathcal{E}), & \mathcal{E} \leq \mathcal{E}_{\text {c-edge }} \\ P_{\mathrm{NM}}(\mathcal{E}), & \mathcal{E}_{\mathrm{c}-\text { edge }} \leq \mathcal{E} \leq \mathcal{E}_{\mathrm{tr}} \\ \left(\frac{v_{\mathrm{s}}}{c}\right)^{2}\left(\mathcal{E}-\mathcal{E}_{\mathrm{tr}}\right)+P_{\mathrm{NM}}\left(\mathcal{E}_{\mathrm{tr}}\right), & \mathcal{E}_{\mathrm{tr}} \leq \mathcal{E}\end{cases}
$$


where $P$ and $\mathcal{E}$ are the pressure and energy density, respectively, and $\mathcal{E}_{\text {tr }}$ is the transition energy density. In region $\mathcal{E} \leq \mathcal{E}_{\mathrm{c}-\text { edge, }}$, we employed the equation of Feynman et al. [19] and Baym et al. [20] for the crust and low densities of neutron star. In the intermediate region, $\mathcal{E}_{\mathrm{c}-\text { edge }} \leq \mathcal{E} \leq \mathcal{E}_{\mathrm{tr}}$, we used a specific EoS based on the MDI model and data from Akmal et al. [21], while for $\mathcal{E}_{\mathrm{tr}} \leq \mathcal{E}$, the EoS is maximally stiff with the speed of sound, defined as $v_{s}=c \sqrt{(\partial P / \partial \mathcal{E})_{\mathrm{S}}}$ (where $S$ is the entropy). In the part of the work concerning the two observed events, the speed of sound is fixed on the two values, $c / \sqrt{3}$ and $c$. The cases which took effect in this study were the ones where the fiducial baryon transition density is $n_{\mathrm{tr}}=p n_{0}$, where $n_{0}$ is the saturation density of symmetric nuclear matter $\left(n_{0}=0.16 \mathrm{fm}^{-3}\right)$ and $p$ takes the values $1,1.5,2,3$. Hence, the predicted EoSs are functional of $n_{\mathrm{tr}}$ and $v_{s}$. For the hypothetical scenario of a non-rotating neutron star with mass equal to the lighter component of the GW190814 event, we used eight values for the speed of sound and two transition densities.

We notice that in the approach of the Eq. (1) the continuity on the EoS is well ensured, but its artificial character does not ensure the continuity in the speed of sound at the transition density. The speed of sound is a quantity that must be treated carefully, especially in regions of discontinuity [22]. In order to ensure continuity and a smooth phase transition, we applied the following parametrization for the speed of sound (for more details see Ref. [23])

$$
\frac{v_{\mathrm{s}}}{c}=\left(a-c_{1} \exp \left[-\frac{\left(n-c_{2}\right)^{2}}{w^{2}}\right]\right)^{1 / 2}, \quad a=1,1 / 3
$$

where the parameters $c_{1}, c_{2}$, and $w$ are fit to the speed of sound and its derivative at $n_{\mathrm{tr}}$, and also to the demands $v_{\mathrm{s}}\left(n_{\mathrm{tr}}\right)=[c, c / \sqrt{3}]$ [18]. We notice that we treated the speed of sound $v_{\mathrm{S}}$ as a constant for the fixed values that we used. Using Eq. (2), the EoS for $n \geq n_{\text {tr }}$ can be constructed with the help of the following recipe [23]

$$
\begin{gathered}
\mathcal{E}_{i+1}=\mathcal{E}_{i}+\Delta \mathcal{E}, \quad P_{i+1}=P_{i}+\left(\frac{v_{s}}{c}\left(n_{i}\right)\right)^{2} \Delta \mathcal{E} \\
\Delta \mathcal{E}=\Delta n\left(\frac{\mathcal{E}_{i}+P_{i}}{n_{i}}\right) \\
\Delta n=n_{i+1}-n_{i} .
\end{gathered}
$$

\subsection{Tidal deformability}

The gravitational waves from the final stages of inspiraling binary neutron stars are a very important source for ground-based gravitational waves detectors [22, 24, 25]. As Flanagan and Hinderer [24] articulated, the tidal effects can be measured during the early part of the evolution when the waveform is relatively clean. The response of a component neutron star in a binary neutron star 
system to the tidal field is described by the tidal Love number $k_{2}$, which relates the quadrupole moment $Q_{i j}$ to the applied tidal field $E_{i j}$ [24]

$$
Q_{i j}=-\frac{2}{3} k_{2} \frac{R^{5}}{G} E_{i j} \equiv-\lambda E_{i j},
$$

where $R$ is the neutron star radius and $\lambda=2 R^{5} k_{2} / 3 G$ is the tidal deformability. The tidal Love number $k_{2}$ is given by $[24,25]$

$$
\begin{aligned}
k_{2} & =\frac{8 \beta^{5}}{5}(1-2 \beta)^{2}\left[2-y_{R}+\left(y_{R}-1\right) 2 \beta\right] \\
& \times\left[2 \beta\left(6-3 y_{R}+3 \beta\left(5 y_{R}-8\right)\right)\right. \\
& +4 \beta^{3}\left(13-11 y_{R}+\beta\left(3 y_{R}-2\right)+2 \beta^{2}\left(1+y_{R}\right)\right) \\
& +3(1-2 \beta)^{2} \\
& \left.\times\left[2-y_{R}+2 \beta\left(y_{R}-1\right)\right] \ln (1-2 \beta)\right]^{-1}
\end{aligned}
$$

where $\beta=G M / R c^{2}$ is the compactness of the neutron star. The parameter $y_{R}$ is determined by solving the corresponding differential equation of $y(r)$ simultaneously with the Tolman-Oppenheimer-Volkoff (TOV) equations [22].

Regarding the binary neutron star systems, one of the binary quantities that is well measured by the gravitational waves detectors is the chirp mass $\mathcal{M}_{c}$, which is a combination of the component masses $[11,13]$

$$
\mathcal{M}_{c}=\frac{\left(m_{1} m_{2}\right)^{3 / 5}}{\left(m_{1}+m_{2}\right)^{1 / 5}}=m_{1} \frac{q^{3 / 5}}{(1+q)^{1 / 5}},
$$

where $m_{1}$ is the mass of the heavier component star and $m_{2}$ is the lighter's one. Hence, the binary mass ratio $q=m_{2} / m_{1}$ lies within $0 \leq q \leq 1$.

The information regarding the tidal effects of the system is imprinted in the gravitational-wave signal This information is characterized by the effective tidal deformability $[11,13]$

$$
\tilde{\Lambda}=\frac{16}{13} \frac{(12 q+1) \Lambda_{1}+(12+q) q^{4} \Lambda_{2}}{(1+q)^{5}},
$$

where $q$ is the mass asymmetry. Moreover, $\Lambda_{i}$ is the dimensionless deformability defined as $[11,13]$

$$
\Lambda_{i}=\frac{2}{3} k_{2}\left(\frac{R_{i} c^{2}}{M_{i} G}\right)^{5} \equiv \frac{2}{3} k_{2} \beta_{i}^{-5}, \quad i=1,2 .
$$

By substituting in Eq. (10) the value of $k_{2}$ from Eq. (7), one can observe that $\Lambda_{i}$ depends both on the compactness of the star as well as on the value of $y(R)$. 


\section{Results}

Firstly, we used two cases for the speed of sound bound; the lower bound of $\left(v_{s} / c\right)^{2}=1 / 3$ and the upper one of $\left(v_{s} / c\right)^{2}=1$. Also, we chose four transition densities, especially $n_{\mathrm{tr}}=\{1,1.5,2,3\} n_{0}$ [15]. Figure 1 shows the mass-radius diagram, after the numerical solution of the TOV equations. In general, the purple-colored curves correspond to the $\left(v_{s} / c\right)^{2}=1 / 3$ bound, while the green ones correspond to the $\left(v_{s} / c\right)^{2}=1$ bound. As one can see, each value of transition density leads to two distinct branches, with the lower (upper) bound $\left(v_{s} / c\right)^{2}=1 / 3\left(\left(v_{s} / c\right)^{2}=1\right)$ of the speed of sound corresponding to lower (higher) masses. As the transition density is getting higher, the EoS is becoming softer. The lower bound of the speed of sound $\left(v_{s} / c\right)^{2}=1 / 3$ leads to a more soft EoS, compared with the $\left(v_{s} / c\right)^{2}=1$ limit. Moreover, the observations of NICER and GW170817 events are in accordance as they show a significant overlap between them. The accepted region of the GW170817 event leads to the exclusion of the EoSs with transition density $n_{\mathrm{tr}}=n_{0}$, for both bounds of the speed of sound.

In our study we used the measured upper limit of the effective tidal deformability $\tilde{\Lambda}$, provided by the events GW170817 and GW190425 [12-14]. The constrained

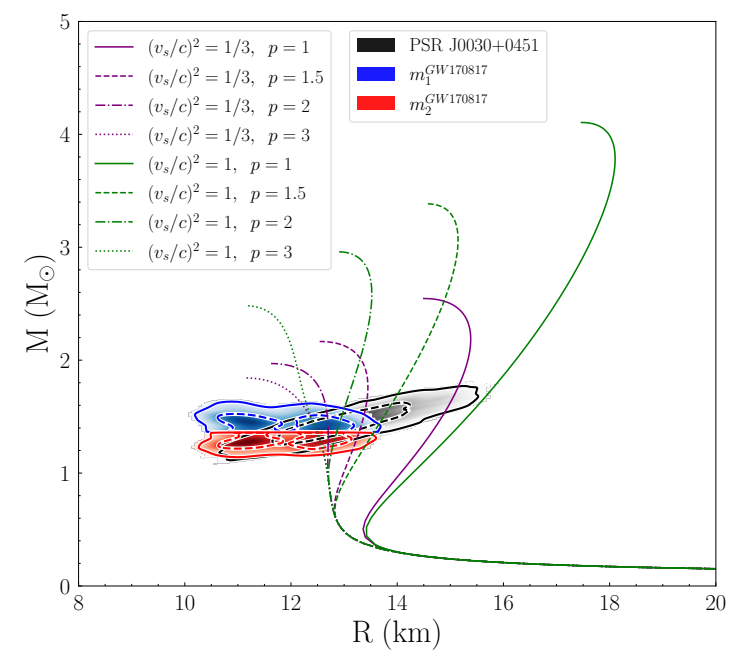

Figure 1. (Color online) Mass-radius diagram for an isolated and non-rotating neutron star, for both speed of sound bounds. The green (purple) curves correspond to the upper (lower) bound of the speed of sound. The black diagonal shaded region indicates the NICER's observation (data taken from Ref. [26]), while the blue upper (red lower) shaded region indicates the heavier (lighter) component of the GW170817 event (data retrieved from Ref. [12]). The solid (dashed) contour boundaries describe the $90 \%$ (50\%) confidence interval. 

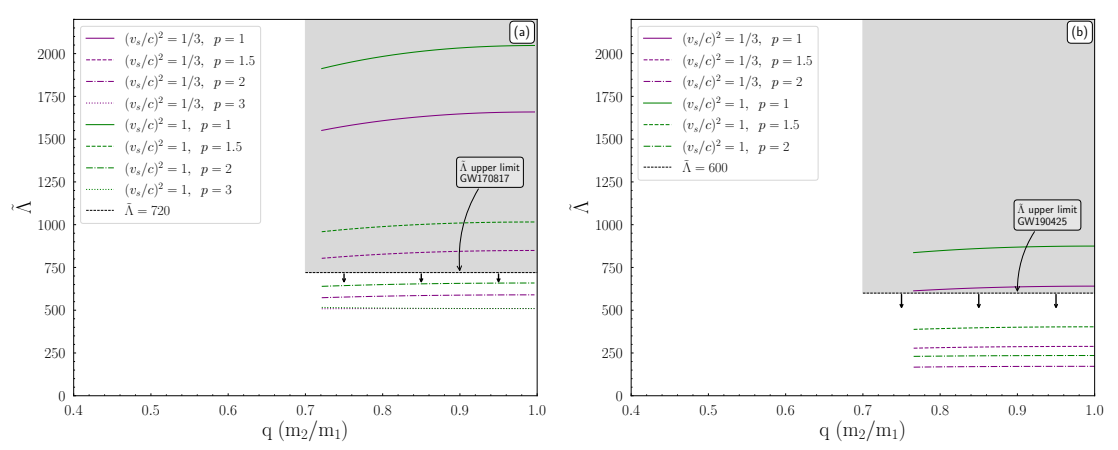

Figure 2. (Color online) The effective tidal deformability $\tilde{\Lambda}$ as a relation of the binary mass ratio $q$ for the event (a) GW170817 and (b) GW190425. The observational upper limits for $\tilde{\Lambda}$ are also indicated, with the grey shaded region indicating the excluded area. The purple (green) curves correspond to the $\left(v_{s} / c\right)^{2}=1 / 3\left(\left(v_{s} / c\right)^{2}=1\right)$ speed of sound bound.

value of the chirp mass for the two events is $\mathcal{M}_{c}=1.186 M_{\odot}$ [13] and $\mathcal{M}_{c}=$ $1.44 M_{\odot}$ [14], respectively. The component masses vary in the ranges $m_{1} \in$ $(1.36,1.60) \quad M_{\odot}$ and $m_{2} \in(1.16,1.36) \quad M_{\odot}$ [13] (GW170817) and $m_{1} \in$ $(1.654,1.894) M_{\odot}$ and $m_{2} \in(1.45,1.654) M_{\odot}(\mathrm{GW} 190425)$. We underline that we modified the range of the component masses (particularly in the second event) to have an equal-mass boundary ( $q \leq 1)$.

Figure 2 shows the effective tidal deformability $\tilde{\Lambda}$ as a function of the binary mass ratio $q$ for both events. The upper limit on $\tilde{\Lambda}$ in Figure 2(a), derived from the event GW170817, excludes for both bounds of the speed of sound the transition densities $n_{\mathrm{tr}}=1,1.5 n_{0}$. By comparing to the previous Figure 1 , the constraints on the upper limit of $\tilde{\Lambda}$ provide further information. Moreover, for the second event in Figure 2(b), a general behavior is that all the EoSs are shifted downwards, because of the higher value of the chirp mass of the binary system. Unfortunately, the upper limit on $\tilde{\Lambda}$, provided by GW190425 event, excludes only the EoSs with transition density $n_{\mathrm{tr}}=n_{0}$, for both speed of sound bounds. In addition, for both events the EoSs with higher values of transition density $n_{\mathrm{tr}}$ provide lower values of $\tilde{\Lambda}$. Hence, the constraints on the upper limit of $\tilde{\Lambda}$ derived from gravitational waves events favor softer EoSs. We notice that for the GW190425 event, we removed from our calculations the cases with transition density $n_{\mathrm{tr}}=3 n_{0}$ because of the fact that the EoS with $\left(v_{s} / c\right)^{2}=1 / 3$ and $n_{\mathrm{tr}}=3 n_{0}$ can not predict the component masses of this event.

Figure 3 displays the behavior of the effective tidal deformability $\tilde{\Lambda}$ related to the transition density $n_{\mathrm{tr}}$ at the maximum mass configuration for both speed of sound bounds, $v_{s}=c / \sqrt{3}$ and $v_{s}=c$, and the two events GW170817 (Figure 3(a)) and GW190425 (Figure 3(b)). According to our findings, by using the measured upper limits on $\tilde{\Lambda}$, for the GW170817 event the lower limit for the 

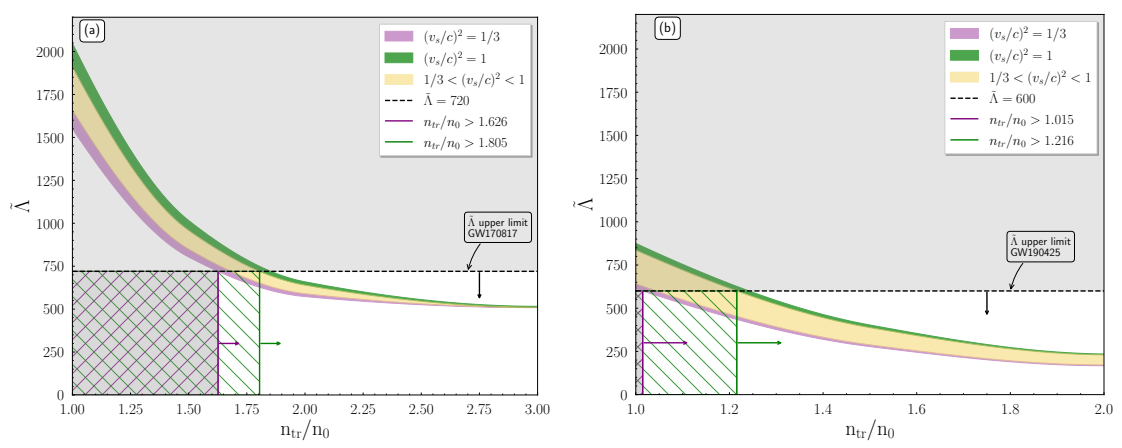

Figure 3. (Color online) $\tilde{\Lambda}$ as a function of the transition density $n_{\mathrm{tr}}$ for both speed of sound bounds and for the event (a) GW170817 (left panel) and (b) GW190425 (right panel). The measured upper limits for $\tilde{\Lambda}[13,14]$ as well as the corresponding lower values of transition density are also indicated. The purple (green) arrow shows the accepted region of transition density for the $v_{s}=c / \sqrt{3}\left(v_{s}=c\right)$ bound. The purple lower (green upper) shaded region indicates the $v_{s}=c / \sqrt{3}\left(v_{s}=c\right)$ speed of sound bound. The yellow shaded area indicates the intermediate values between the two speed of sound bounds.

transition density is $1.626 n_{0}$ for $v_{s}=c / \sqrt{3}$ and $1.805 n_{0}$ for $v_{s}=c$. For the second event GW190425 the corresponding limits are $1.015 n_{0}$ for $v_{s}=c / \sqrt{3}$ and $1.216 n_{0}$ for $v_{s}=c$. Hence, the first event provides more stringent constraints on the EoS compared to the second event. In addition, the result for the bound $v_{s}=c / \sqrt{3}$ is in accordance with other studies (see Ref. [27]). For further details, we refer to our work [15].

The recent detection of the GW190814 event, in which the identity of the lighter component is a mystery, began a very interesting discussion about the nature of this compact object with mass $\sim 2.6 M_{\odot}$. At this part, we extended our previous work aiming to study what would mean the existence of such a massive nonrotating neutron star. In our hypothetical scenario, we focused on two transition densities $n_{\mathrm{tr}}=[1.5,2] n_{0}$ and eight values of speed of sound bounds $\left(v_{s} / c\right)^{2}=$ $[1 / 3,0.4,0.5,0.6,0.7,0.8,0.9,1][17]$. We chose these values for the transition density to be close to the constraints of Ref. [15].

Figure 4(a) shows the corresponding mass-radius diagram. One can observe the two main branches of EoSs, related to each value of transition density. The solid (dashed) curves correspond to the $n_{\mathrm{tr}}=1.5 n_{0}\left(n_{\mathrm{tr}}=2 n_{0}\right)$ value. Across the same value of $n_{\mathrm{tr}}$, the lighter curves correspond to higher values of the speed of sound. The blue solid horizontal line indicates the estimation of the recently observed massive compact object of Ref. [16]. To be more specific, the EoSs with $n_{\mathrm{tr}}=1.5 n_{0}$ are stiffer comparing to those with $n_{\mathrm{tr}}=2 n_{0}$. In addition, the EoSs with higher speed of sound bounds provide higher values of neutron star mass. Hence, a high enough value for the speed of sound is needed for such a 

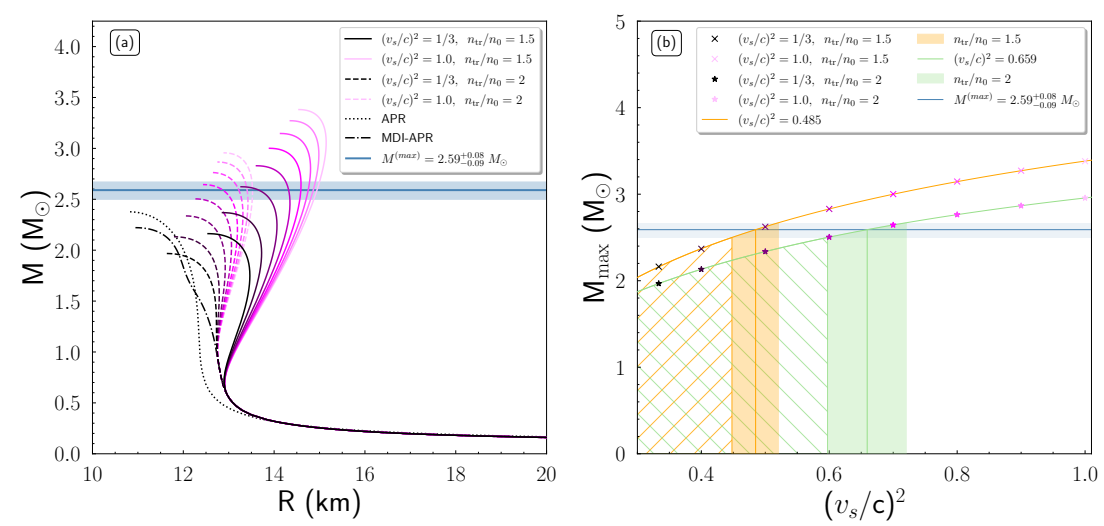

Figure 4. (Color online) (a) Mass vs. radius for an isolated non-rotating neutron star. The darker purple curves' color corresponds to the lower values of speed of sound. The blue horizontal line and region show the mass estimation of the massive compact object of Ref. [16]. (b) The maximum mass $M_{\max }$ of a non-rotating neutron star as a relation to the speed of sound bounds $\left(v_{s} / c\right)^{2}$. The orange (green) vertical line shows the corresponding value of the speed of sound for a massive object with $M=2.59 M_{\odot}$.

massive neutron star.

Figure 4(b) shows the trend that is present in the maximum mass values. The cross (star) marks indicate the maximum masses of $n_{\mathrm{tr}}=1.5 n_{0}\left(n_{\mathrm{tr}}=2 n_{0}\right)$. The blue solid horizontal line shows the estimation of the recently observed massive compact object of Ref. [16]. By applying a fitting formula, one can estimate the specific value of the speed of sound that is needed for the description of such a massive neutron star. Particularly, the speed of sound must be (a) $\left(v_{s} / c\right)^{2}=0.485\left(n_{\mathrm{tr}}=1.5 n_{0}\right)$, and (b) $\left(v_{s} / c\right)^{2}=0.659\left(n_{\mathrm{tr}}=2 n_{0}\right)$. Therefore, a very massive non-rotating neutron star with $M=2.59 M_{\odot}$ requires high enough value for the speed of sound (higher than the conformal limit $v_{s}=c / \sqrt{3}$ ).

\section{Conclusions}

Firstly, we studied the EoS, and especially imposed constraints on the speed of sound (which affects the stiffness of the EoS) and the transition density by using the recent observations of two binary neutron stars mergers (GW170817 and GW190425 events). The implemented method that we developed was based on the upper limits of the effective tidal deformability (derived from the mentioned events), combined with measurements of the maximum neutron star mass. As a base in our study we used the APR1-MDI EoS, for two cases of the speed of sound bounds; the conformal case $v_{s}=c / \sqrt{3}$ and the causal one of $v_{s}=c$. 


\section{Constraints on the equation of state}

The treatment of the effective tidal deformability as a function of the transition density allowed us to extract constraints on the speed of sound bounds. Specifically, for the GW170817 event we found that the speed of sound must be lower than the value $v_{s}=c / \sqrt{3}$ at least up to densities $n_{\mathrm{tr}} \approx 1.6 n_{0}$ and lower than $v_{s}=c$ up to densities $n_{\mathrm{tr}} \approx 1.8 n_{0}$. For the GW190425 event, the respective values are $n_{\mathrm{tr}} \approx n_{0}$ for the lower speed of sound bound and $n_{\mathrm{tr}} \approx 1.2 n_{0}$ for the upper one. These constraints are less rigorous than those derived from the GW170817 event.

Regarding the GW190814 event, we investigated the hypothetical scenario of a non-rotating neutron star with $M=2.59_{-0.09}^{+0.08} M_{\odot}$. In that case, by studying the maximum mass of each EoS as a function of the speed of sound, we obtained a specific value for each transition density. In particular, the $n_{\mathrm{tr}}=1.5 n_{0}$ case leads to $\left(v_{s} / c\right)^{2} \in[0.448,0.52]$ while the $n_{\mathrm{tr}}=2 n_{0}$ case leads to $\left(v_{s} / c\right)^{2} \in$ $[0.597,0.72]$. Therefore, the existence of such a massive neutron star should lead to the violation of the conformal limit of $\left(v_{s} / c\right)^{2}=1 / 3$. This hypothetical scenario demonstrates the significant impact of the speed of sound, as it affects dramatically the EoS (in combination with the transition density).

\section{Acknowledgements}

The authors thank the Bulgarian National Science Fund (BNSF) for its support under contract KP-06-N28/6.

\section{References}

[1] S.L. Shapiro, S.A. Teukolsky (1983) "Black Holes, White Dwarfs, and Neutron Stars". (John Wiley and Sons, New York).

[2] Ya. B. Zel'dovich (1962) Sov. Phys. JETP 141143.

[3] J.B. Hartle (1978) Phys. Rep. 46201.

[4] P. Bedaque, A.W. Steiner (2015) Phys. Rev. Lett. 114031103.

[5] C.C. Moustakidis, T. Gaitanos, C. Margaritis, and G.A. Lalazissis (2017) Phys. Rev. C 95045801.

[6] B. Reed, and C.J. Horowitz (2020) Phys. Rev. C 101045803.

[7] E.D. Van Oeveren, and J.L. Friedman (2017) Phys. Rev. D 95083014.

[8] Y.-L. Ma, and M. Rho (2019) Phys. Rev. D 100114003.

[9] G. Baym et al. (2018) Rept. Prog. Phys. 81056902.

[10] E. Annala, T. Gorda, A. Kurkela, J. Nattila, and A. Vuorinen (2020) Nat. Phys. 16 907.

[11] B.P. Abbott et al. (2017) Phys. Rev. Lett. 119161101.

[12] B.P. Abbott et al. (2018) Phys. Rev. Lett. 121161101.

[13] B.P. Abbott et al. (2019) Phys. Rev. X 9011001.

[14] B.P. Abbott et al. (2020) Astrophys. J. Lett. 892 L3.

[15] A. Kanakis-Pegios, P.S. Koliogiannis, and C.C. Moustakidis (2020) Phys. Rev. C 1020558013. 
[16] R. Abbott et al. (2020) Astrophys. J. 896 L44.

[17] A. Kanakis-Pegios, P. S. Koliogiannis, and C. C. Moustakidis (2021) Symmetry 14 183.

[18] Ch. Margaritis, P. S. Koliogiannis, and Ch. C. Moustakidis (2020) Phys. Rev. D 101 043023.

[19] R. Feynman, N. Metropolis, and E. Teller (1949) Phys. Rev. 751561.

[20] G. Baym, C. Pethik, and P. Sutherland (1971) Astrophys. J. 170299.

[21] A. Akmal, V.R. Pandharipande, and D.G. Ravenhall (1998) Phys. Rev. C 581804.

[22] S. Postnikov, M. Prakash, and J.M. Lattimer (2010) Phys. Rev. D 82024016.

[23] I. Tews, J. Carlson, S. Gandolfi, and S. Reddy (2018) Astrophys. J. 860149.

[24] E.E. Flanagan and T. Hinderer (2008) Phys. Rev. D 77 021502(R).

[25] T. Hinderer (2008) Astrophys. J. 6771216.

[26] M.C. Miller et al. (2019) Astrophys. J. Lett. 887 L24.

[27] S.K. Greif, G. Raaijmakers, K. Hebeler, A. Schwenk, and A.L. Watts (2019) Mon. Not. Roy. Astron. Soc. 4855363. 